\title{
THE QUANTUM LEFSCHETZ HYPERPLANE PRINCIPLE CAN FAIL FOR POSITIVE ORBIFOLD HYPERSURFACES
}

\author{
Tom Coates, Amin Gholampour, Hiroshi Iritani, Yunfeng Jiang, \\ Paul Johnson and Cristina Manolache
}

\begin{abstract}
We show that the Quantum Lefschetz Hyperplane Principle can fail for certain orbifold hypersurfaces and complete intersections. It can fail even for orbifold hypersurfaces defined by a section of an ample line bundle.
\end{abstract}

\section{Introduction}

Let $X$ be a projective algebraic variety. Let $g$ and $n$ be non-negative integers, $d$ be an element of $H_{2}(X ; \mathbb{Z})$, and $X_{g, n, d}$ be the moduli stack of degree- $d$ stable maps to $X$ from genus- $g$ curves with $n$ marked points [12]. Gromov-Witten invariants of $X$ are intersection numbers in $X_{g, n, d}$ against the virtual fundamental cycle $\left[X_{g, n, d}\right]^{\text {vir }}[2,14]$. Let $Y \subset X$ be a complete intersection cut out by a section of a vector bundle $E \rightarrow X$ which is the direct sum of line bundles $E=\oplus E_{j}$. The inclusion $i: Y \rightarrow X$ induces a morphism of moduli stacks $\iota: Y_{g, n, \delta} \rightarrow X_{g, n, i_{\star} \delta}$. Suppose that the line bundles $E_{j}$ each satisfy the positivity condition:

$(*) \quad c_{1}\left(E_{j}\right) \cdot d \geq 0$ whenever $d$ is the degree of genus-zero stable map to $X$

Then:

$$
\sum_{\delta: i_{\star} \delta=d} \iota_{\star}\left[Y_{0, n, \delta}\right]^{\mathrm{vir}}=\left[X_{0, n, d}\right]^{\mathrm{vir}} \cap \boldsymbol{e}\left(E_{0, n, d}\right),
$$

where $\boldsymbol{e}$ is the Euler class and $E_{0, n, d}$ is a certain vector bundle on $X_{0, n, d}$, described in Section 5 below. Equality $(\dagger)$ lies at the heart of the Quantum Lefschetz Hyperplane Principle, and hence of the proof of mirror symmetry for toric complete intersections $[6,7,16-18]$. (See [5] for a very clear explanation of this.)

In this paper, we show by means of examples that, for orbifold complete intersections, $(*)$ does not imply $(\dagger)$. We give examples of smooth orbifolds $X$ and complete intersections $Y \subset X$ cut out by sections of vector bundles $E=\oplus E_{j} \rightarrow X$ such that each $E_{j}$ is a line bundle that satisfies $(*)$ but there is no cohomology class $e$ on $X_{0, n, d}$ with:

$$
\sum_{\delta: i_{\star} \delta=d} \iota_{\star}\left[Y_{0, n, \delta}\right]^{\mathrm{vir}}=\left[X_{0, n, d}\right]^{\mathrm{vir}} \cap e .
$$

In particular, there is no vector bundle $E_{0, n, d}$ on $X_{0, n, d}$ such that ( $\dagger$ ) holds. Thus the Quantum Lefschetz Hyperplane Principle, as currently understood, can fail for positive orbifold complete intersections.

Received by the editors November 09, 2011.

Key words and phrases. Gromov-Witten invariants, orbifolds, quantum cohomology, hypersurfaces, complete intersections, Quantum Lefschetz Hyperplane Theorem. 
Remark 1. There does not seem to be a universally accepted definition of ampleness for line bundles on orbifolds (see [20, Section 2.5] for one possibility), but any reasonable definition will imply property $(*)$.

\section{Genus-one Gromov-Witten invariants of the quintic threefold}

It is well known that there is no straightforward analog of the Quantum Lefschetz Hyperplane Principle for higher-genus stable maps (those with $g>0$ ), even when both $X$ and $Y$ are smooth varieties. Givental [8] gave an example that demonstrates this; for the convenience of the reader we repeat his argument here. Recall that for any smooth projective variety $X$, the moduli stack $X_{1,1,0}$ of degree-zero one-pointed stable maps from genus-one curves to $X$ is isomorphic to $X \times \overline{\mathcal{M}}_{1,1}$. Let $\pi_{1}: X_{1,1,0} \rightarrow X$ denote projection to the first factor, $\pi_{2}: X_{1,1,0} \rightarrow \overline{\mathcal{M}}_{1,1}$ denote projection to the second factor, and $\psi_{1} \in H^{2}\left(\overline{\mathcal{M}}_{1,1}\right)$ denote the universal cotangent line class. The virtual fundamental class is:

$$
\left[X_{1,1,0}\right]^{\mathrm{vir}}=\left[X_{1,1,0}\right] \cap\left(\pi_{1}^{\star}\left(c_{D}(T X)\right)-\pi_{1}^{\star}\left(c_{D-1}(T X)\right) \cup \pi_{2}^{\star}\left(\psi_{1}\right)\right),
$$

where $D$ is the complex dimension of $X$.

Now let $X=\mathbb{P}^{4}$ and $Y \subset X$ be a quintic threefold, i.e., $Y$ is the hypersurface cut out by a generic section of $E=\mathcal{O}(5) \rightarrow X$. As before, let $i: Y \rightarrow X$ be the inclusion and $\iota: Y_{1,1,0} \rightarrow X_{1,1,0}$ be the induced map of moduli stacks. We will show that there is no cohomology class $e$ on $X_{1,1,0}$ such that:

$$
\iota_{\star}\left[Y_{1,1,0}\right]^{\mathrm{vir}}=\left[X_{1,1,0}\right]^{\mathrm{vir}} \cap e
$$

Since both $\left[Y_{1,1,0}\right]^{\text {vir }}$ and $\left[X_{1,1,0}\right]^{\text {vir }}$ have the same dimension, this amounts to showing that $\iota_{\star}\left[Y_{1,1,0}\right]^{\text {vir }}$ is not a scalar multiple of $\left[X_{1,1,0}\right]^{\text {vir }}$.

Let $h \in H^{2}(X)$ denote the first Chern class of the line bundle $\mathcal{O}(1)$ on $X$. Applying the total Chern class to both sides of the equality:

$$
T Y \oplus i^{\star} \mathcal{O}(5)=i^{\star} T X
$$

yields $c_{1}(T Y)=0, c_{2}(T Y)=10 i^{\star}\left(h^{2}\right), c_{3}(T Y)=-40 i^{\star}\left(h^{3}\right)$. Thus,

$$
\begin{aligned}
\iota_{\star}\left[Y_{1,1,0}\right]^{\mathrm{vir}} & =\iota_{\star}\left(-40 i^{\star}\left(h^{3}\right)-10 i^{\star}\left(h^{2}\right) \psi_{1}\right) \\
& =\left(-40 h^{3}-10 h^{2} \psi_{1}\right) \cup \iota_{\star} 1 \\
& =-200 h^{4}-50 h^{3} \psi_{1}
\end{aligned}
$$

where in the second line we used the projection formula and in the last line we used the fact that the normal bundle to the inclusion $\iota$ is $\pi_{1}^{\star} \mathcal{O}(5)$. On the other hand,

$$
\left[X_{1,1,0}\right]^{\mathrm{vir}}=5 h^{4}-10 h^{3} \psi_{1}
$$

and so $\iota_{\star}\left[Y_{1,1,0}\right]^{\text {vir }}$ is not a scalar multiple of $\left[X_{1,1,0}\right]^{\text {vir }}$.

\section{A trivial example}

Let $X$ be the orbifold $\mathbb{P}(1,1,2,2)$, and let $Y=\mathbb{P}(1,2,2)$ be the orbifold hypersurface in $X$ defined by the vanishing of a section of $\mathcal{O}(1)$. Let $X_{0, \overrightarrow{4}, 0}$ and $Y_{0, \overrightarrow{4}, 0}$ denote $^{1}$ the

\footnotetext{
${ }^{1}$ The vector $\overrightarrow{4}$ in the subscript here is to emphasize the fact that we specify not only the number of marked points on the curves but also the isotropy group at each marked point.
} 
moduli stacks of genus-zero degree-zero stable maps to (respectively) $X$ and $Y$, from orbicurves with four marked points such that the isotropy group at each marked point is $\mu_{2}$. As before, write $i: Y \rightarrow X$ for the inclusion map, and $\iota: Y_{0, \overrightarrow{4}, 0} \rightarrow X_{0, \overrightarrow{4}, 0}$ for the induced morphism of moduli stacks. We have vdim $X_{0, \overrightarrow{4}, 0}=0$ and $\operatorname{vdim} Y_{0, \overrightarrow{4}, 0}=1$; so for dimensional reasons there is no cohomology class $e$ on $X_{0, \overrightarrow{4}, 0}$ such that:

$$
\iota_{\star}\left[Y_{0, \overrightarrow{4}, 0}\right]^{\mathrm{vir}}=\left[X_{0, \overrightarrow{4}, 0}\right]^{\mathrm{vir}} \cap e
$$

\section{A non-trivial example}

Let $X$ be the orbifold $\mathbb{P}(1,1,1,2,2,2,2)$, and let $Y=\mathbb{P}(1,1,2,2,2)$ be the orbifold complete intersection in $X$ defined by the vanishing of a section of $\mathcal{O}(1) \oplus \mathcal{O}(2)$. Let $X_{0, \overrightarrow{4}, 0}$ and $Y_{0, \overrightarrow{4}, 0}$ denote the moduli stacks of genus-zero degree-zero stable maps to (respectively) $X$ and $Y$, from orbicurves with four marked points such that the isotropy group at each marked point is $\mu_{2}$. Let $i: Y \rightarrow X$ be the inclusion map and $\iota: Y_{0, \overrightarrow{4}, 0} \rightarrow X_{0, \overrightarrow{4}, 0}$ be the induced morphism of moduli stacks. We have:

$$
\operatorname{vdim} X_{0, \overrightarrow{4}, 0}=1, \quad \operatorname{vdim} Y_{0, \overrightarrow{4}, 0}=1
$$

and the coarse moduli spaces are:

$$
\left|X_{0, \overrightarrow{4}, 0}\right|=\mathbb{P}^{3} \times \overline{\mathcal{M}}_{0,4}, \quad\left|Y_{0, \overrightarrow{4}, 0}\right|=\mathbb{P}^{2} \times \overline{\mathcal{M}}_{0,4},
$$

where $\overline{\mathcal{M}}_{0,4}$ is Deligne-Mumford space. Recall that the rational homology and cohomology groups of a smooth stack coincide with the rational homology and cohomology groups of the coarse moduli space [1, Section 2]. We therefore regard all virtual fundamental classes, cohomology classes, Chern classes, etc. in our calculation as living on the coarse moduli spaces of the stacks involved.

Proposition 4.1. We have

$$
X_{0, \overrightarrow{4}, 0}=\mathbb{P}(2,2,2,2) \times \overline{\mathcal{M}}_{0,4}, \quad Y_{0, \overrightarrow{4}, 0}=\mathbb{P}(2,2,2) \times \overline{\mathcal{M}}_{0,4} .
$$

Proof. We prove the proposition only for $X_{0, \overrightarrow{4}, 0}$; the argument for $Y_{0, \overrightarrow{4}, 0}$ is almost identical. The moduli stack $X_{0, \overrightarrow{4}, 0}$ is a $\mu_{2}$-gerbe over the coarse moduli space $\left|X_{0, \overrightarrow{4}, 0}\right|$. Such gerbes necessarily have trivial $\operatorname{lien}^{2}$, and thus are classified by the sheaf cohomology group:

$$
\begin{aligned}
H^{2}\left(\left|X_{0, \overrightarrow{4}, 0}\right|, \mu_{2}\right) & \cong H^{2}\left(\mathbb{P}^{3}, \mu_{2}\right) \times H^{2}\left(\overline{\mathcal{M}}_{0,4}, \mu_{2}\right) \\
& \cong \mu_{2} \times \mu_{2} .
\end{aligned}
$$

The gerbe $\mathbb{P}(2,2,2,2) \times \overline{\mathcal{M}}_{0,4}$ over $\mathbb{P}^{3} \times \overline{\mathcal{M}}_{0,4}$ is non-trivial on the first factor and trivial on the second factor, and therefore corresponds to the class $(-1,1) \in \mu_{2} \times \mu_{2}$. It thus suffices to show that the gerbe $X_{0, \overrightarrow{4}, 0}$ over $\mathbb{P}^{3} \times \overline{\mathcal{M}}_{0,4}$ also corresponds to the class $(-1,1) \in \mu_{2} \times \mu_{2}$.

\footnotetext{
${ }^{2}$ The lien of a gerbe is also known as its band. For a careful discussion of bands and the classification of gerbes; see [19, Lecture 3].
} 
Let $\pi_{1}$ and $\pi_{2}$ denote the projections to (respectively) the first and second factors of the product $\mathbb{P}^{3} \times \overline{\mathcal{M}}_{0,4}$. There is a commutative diagram:

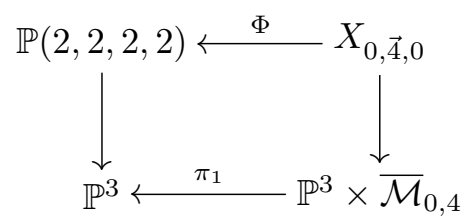

where each vertical arrow is the canonical map from a stack to its coarse moduli space, and $\Phi$ is the natural morphism coming from the fact that $X_{0, \overrightarrow{4}, 0}$ is a moduli stack of degree-zero maps. This implies that restricting the gerbe $X_{0, \overrightarrow{4}, 0}$ over $\mathbb{P}^{3} \times \overline{\mathcal{M}}_{0,4}$ to a fiber of $\pi_{2}$ yields the non-trivial gerbe $\mathbb{P}(2,2,2,2)$ over $\mathbb{P}^{3}$. On the other hand, restricting the gerbe $X_{0, \overrightarrow{4}, 0}$ over $\mathbb{P}^{3} \times \overline{\mathcal{M}}_{0,4}$ to a fiber of $\pi_{1}$ yields the trivial gerbe $\left(B \mu_{2}\right)_{0, \overrightarrow{4}, 0}$ over $\overline{\mathcal{M}}_{0,4}$. Thus, the gerbe $X_{0, \overrightarrow{4}, 0}$ over $\mathbb{P}^{3} \times \overline{\mathcal{M}}_{0,4}$ corresponds to the class $(-1,1) \in \mu_{2} \times \mu_{2}$. The Proposition is proved.

We will show that there is no cohomology class $e$ on $X_{0, \overrightarrow{4}, 0}$ such that:

$$
\iota_{\star}\left[Y_{0, \overrightarrow{4}, 0}\right]^{\mathrm{vir}}=\left[X_{0, \overrightarrow{4}, 0}\right]^{\mathrm{vir}} \cap e .
$$

As before, this amounts to showing that $\iota_{\star}\left[Y_{0, \overrightarrow{4}, 0}\right]^{\text {vir }}$ and $\left[X_{0, \overrightarrow{4}, 0}\right]^{\text {vir }}$ are not scalar multiples of each other. Consider the universal families over the moduli stacks $X_{0, \overrightarrow{4}, 0}$ and $Y_{0, \overrightarrow{4}, 0}$ :
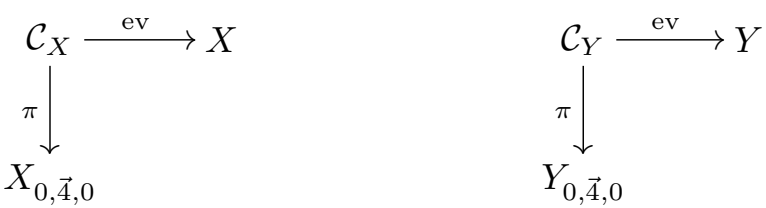

The moduli stack $X_{0, \overrightarrow{4}, 0}$ is smooth, with obstruction bundle $\mathcal{V}^{\oplus 3}$ where:

$$
\mathcal{V}=R^{1} \pi_{\star}\left(\mathrm{ev}^{\star} \mathcal{O}_{X}(1)\right)
$$

Thus, the virtual fundamental class of $X_{0, \overrightarrow{4}, 0}$ is:

$$
\left[X_{0, \overrightarrow{4}, 0}\right]^{\text {vir }}=\left[X_{0, \overrightarrow{4}, 0}\right] \cap \boldsymbol{e}(\mathcal{V})^{3}
$$

The moduli stack $Y_{0, \overrightarrow{4}, 0}$ is also smooth, with obstruction bundle:

$$
\left[R^{1} \pi_{\star}\left(\mathrm{ev}^{\star} \mathcal{O}_{Y}(1)\right)\right]^{\oplus 2}
$$


and since the universal family over $Y_{0, \overrightarrow{4}, 0}$ is the restriction to $Y_{0, \overrightarrow{4}, 0}$ of the universal family over $X_{0, \overrightarrow{4}, 0}$ :

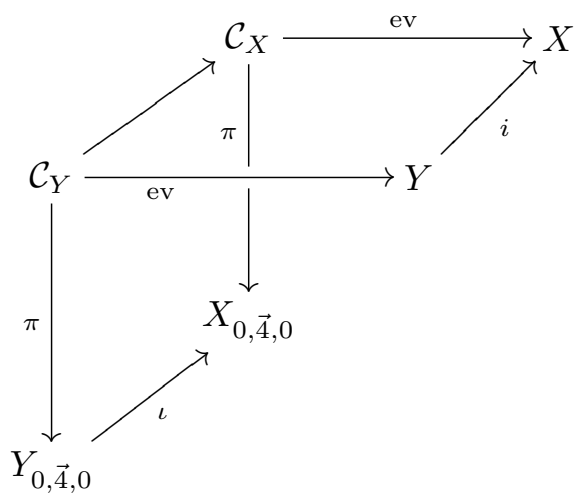

it follows that:

$$
\left[R^{1} \pi_{\star}\left(\mathrm{ev}^{\star} \mathcal{O}_{Y}(1)\right)\right]^{\oplus 2}=\iota^{\star} \mathcal{V}^{\oplus 2}
$$

Thus, the virtual fundamental class of $Y_{0, \overrightarrow{4}, 0}$ is

$$
\left[Y_{0, \overrightarrow{4}, 0}\right]^{\mathrm{vir}}=\left[Y_{0, \overrightarrow{4}, 0}\right] \cap \boldsymbol{e}\left(\iota^{\star} \mathcal{V}\right)^{2} .
$$

We next identify the Euler class of $\mathcal{V}$. As before, let $\pi_{1}$ and $\pi_{2}$ denote the projections to (respectively) the first and second factors of the coarse moduli space $\left|X_{0, \overrightarrow{4}, 0}\right|=$ $\mathbb{P}^{3} \times \overline{\mathcal{M}}_{0,4}$. Let $h \in H^{2}\left(\left|X_{0, \overrightarrow{4}, 0}\right|\right)$ be the pullback along $\pi_{1}$ of the first Chern class of the line bundle $\mathcal{O}(1) \rightarrow \mathbb{P}^{3}$. Let $\psi \in H^{2}\left(\left|X_{0, \overrightarrow{4}, 0}\right|\right)$ be the pullback along $\pi_{2}$ of the universal cotangent line class on $\overline{\mathcal{M}}_{0,4}$ corresponding to the first marked point. Note that $\{h, \psi\}$ forms a basis for $H^{2}\left(\left|X_{0, \overrightarrow{4}, 0}\right|\right)$.

\section{Lemma 4.2.}

$$
\boldsymbol{e}(\mathcal{V})=\frac{1}{2}(h-\psi)
$$

Proof. Consider the universal family:

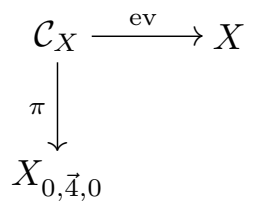

and recall that $X_{0, \overrightarrow{4}, 0} \cong \mathbb{P}(2,2,2,2) \times \overline{\mathcal{M}}_{0,4}$. We have

$$
\begin{aligned}
\mathcal{V} & =-\pi_{\star} \operatorname{ev}^{\star}\left(\mathcal{O}_{X}(1)\right) & & \text { (K-theory pushforward) } \\
& =-\pi_{\star} \pi^{\star}\left(\mathcal{O}_{\mathbb{P}(2,2,2,2)}(1)\right) & & \\
& =\mathcal{O}_{\mathbb{P}(2,2,2,2)}(1) \otimes\left(-\pi_{\star} \mathcal{O}_{\mathcal{C}_{X}}\right) & & \text { (projection formula). }
\end{aligned}
$$

We saw in the proof of Proposition 4.1 that restricting the gerbe $X_{0, \overrightarrow{4}, 0}$ over $\mathbb{P}^{3} \times \overline{\mathcal{M}}_{0,4}$ to a fiber of $\pi_{2}$ yields $\mathbb{P}(2,2,2,2)$. The restriction of $\mathcal{V}$ to this copy of $\mathbb{P}(2,2,2,2)$ is $\mathcal{O}_{\mathbb{P}(2,2,2,2)}(1)$, and so:

$$
\boldsymbol{e}(\mathcal{V})=\frac{1}{2} h+\alpha \psi
$$


for some scalar $\alpha$. We saw in the proof of Proposition 4.1 that restricting the gerbe $X_{0, \overrightarrow{4}, 0}$ over $\mathbb{P}^{3} \times \overline{\mathcal{M}}_{0,4}$ to a fiber of $\pi_{1}$ yields $\left(B \mu_{2}\right)_{0, \overrightarrow{4}, 0}$. The restriction of $\mathcal{V}$ to this copy of $\left(B \mu_{2}\right)_{0, \overrightarrow{4}, 0}$ is $\mathbb{E}^{\vee}$, where $\mathbb{E}$ is the Hodge bundle on $\left(B \mu_{2}\right)_{0, \overrightarrow{4}, 0}$, and so we can determine the scalar $\alpha$ by comparing the integrals:

$$
\int_{\left(B \mu_{2}\right)_{0, \overrightarrow{4}, 0}} c_{1}(\mathbb{E})=\frac{1}{4}, \quad \quad \int_{\overline{\mathcal{M}}_{0,4}} \psi_{1}=1 .
$$

The right-hand integral here is well known; the left-hand integral is computed in [10, Section 3.1].

Proposition 4.3. The classes $\iota_{\star}\left[Y_{0, \overrightarrow{4}, 0}\right]^{\mathrm{vir}}$ and $\left[X_{0, \overrightarrow{4}, 0}\right]^{\mathrm{vir}}$ are not scalar multiples of each other.

Proof. Combining (4.2) and (4.3) with Lemma 4.2, we have:

$$
\left[X_{0, \overrightarrow{4}, 0}\right]^{\text {vir }}=\left[X_{0, \overrightarrow{4}, 0}\right] \cap\left(\frac{1}{8} h^{3}-\frac{3}{8} h^{2} \psi\right)
$$

and

$$
\begin{aligned}
\iota_{\star}\left[Y_{0, \overrightarrow{4}, 0}\right]^{\text {vir }} & =\iota_{\star}\left[Y_{0, \overrightarrow{4}, 0}\right] \cap \boldsymbol{e}(\mathcal{V})^{2} \\
& =\left[X_{0, \overrightarrow{4}, 0}\right] \cap\left(h \cup \frac{1}{4}(h-\psi)^{2}\right) \\
& =\left[X_{0, \overrightarrow{4}, 0}\right] \cap\left(\frac{1}{4} h^{3}-\frac{1}{2} h^{2} \psi\right) .
\end{aligned}
$$

Since $h^{3}$ and $h^{2} \psi$ are linearly independent in $H^{6}\left(\left|X_{0, \overrightarrow{4}, 0}\right|\right)$, the Proposition follows.

\section{Convexity}

Our examples show that the key property underlying $(\dagger)$ is not positivity $(*)$ of $E$ but rather convexity of $E$. Recall that a vector bundle $E \rightarrow X$ is called convex if and only if $H^{1}\left(C, f^{\star} E\right)=0$ for all stable maps $f: C \rightarrow E$ from genus-zero (orbi)curves. Suppose that $E=\oplus_{j} E_{j}$ is a direct sum of line bundles and that each line bundle $E_{j}$ satisfies $(*)$. If $X$ is a smooth variety then $E$ is automatically convex but, as we will discuss below, this need not be the case if $X$ is an orbifold.

Let $X$ be a smooth projective variety or smooth orbifold, and let $E \rightarrow X$ be a convex vector bundle. Let:

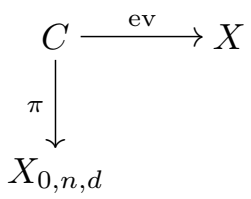

be the universal family over the moduli stack $X_{0, n, d}$ of genus-zero stable maps and let $E_{0, n, d}=R^{0} \pi_{\star} \mathrm{ev}^{\star} E$. Convexity implies that $R^{1} \pi_{\star} \mathrm{ev}^{\star} E=0$, and hence that $E_{0, n, d}$ is a vector bundle on $X_{0, n, d}$.

Proposition 5.1 (Convexity implies $(\dagger)$ ). Let $X$ be a smooth projective variety or orbifold, let $E \rightarrow X$ be a convex vector bundle, and let $Y$ be the subvariety or suborbifold of $X$ cut out by a generic section s of $E$. Let $i: Y \rightarrow X$ be the inclusion map, and let $\iota: Y_{0, n, \delta} \rightarrow X_{0, n, i_{\star} \delta}$ be the induced morphism of moduli stacks. Then:

$$
\sum_{\delta: i_{\star} \delta=d} \iota_{\star}\left[Y_{0, n, \delta}\right]^{\mathrm{vir}}=\left[X_{0, n, d}\right]^{\mathrm{vir}} \cap \boldsymbol{e}\left(E_{0, n, d}\right) .
$$


Proof. The stacks $X_{0, n, d}$ and $Y_{0, n, \delta}$ carry perfect obstruction theories relative to the Artin stack $\mathfrak{M}$ of marked twisted curves [1]:

$$
\begin{aligned}
& \left(R^{\bullet} \pi_{\star} \mathrm{ev}^{\star} T X\right)^{\vee} \text { for } X_{0, n, d} \\
& \left(R^{\bullet} \pi_{\star} \mathrm{ev}^{\star} T Y\right)^{\vee} \text { for } Y_{0, n, \delta} .
\end{aligned}
$$

Write:

$$
Y_{d}=\coprod_{\delta: i_{\star} \delta=d} Y_{0, n, \delta}
$$

and consider the 2-Cartesian digram of Deligne-Mumford stacks:

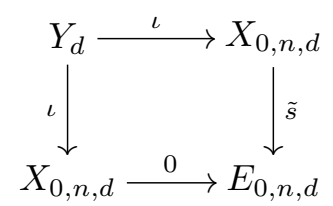

where 0 is the zero section of $E_{0, n, d}$ and $\tilde{s}$ is the section of $E_{0, n, d}$ induced by $s$. For a morphism $A \rightarrow B$ of stacks, let $L_{A / B}$ denote the relative cotangent complex [13]. There is a morphism of distinguished triangles in the derived category of sheaves on $Y_{d}$ :

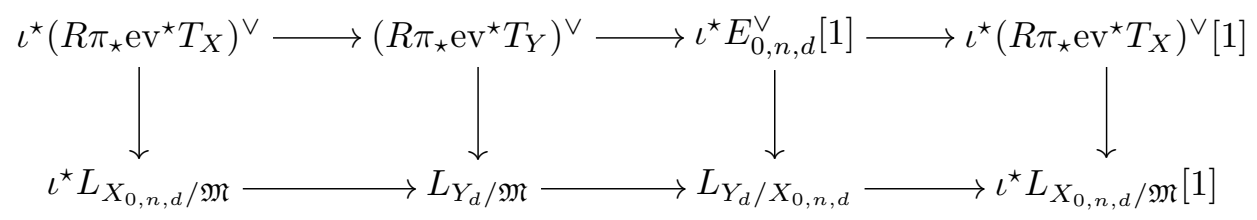

and, since $E$ is convex, we have:

$$
\iota^{\star} E_{0, n, d}^{\vee}[1]=\iota^{\star} L_{X_{0, n, d} / E_{0, n, d}} .
$$

Thus the perfect obstruction theories (5.1) are compatible over $\iota: Y_{d} \rightarrow X_{0, n, d}$ in the sense of Behrend and Fantechi [2, Definition 5.8]. Functoriality for the virtual fundamental class [11] now implies that:

$$
0^{!}\left[X_{0, n, d}\right]^{\mathrm{vir}}=\sum_{\delta: i_{\star} \delta=d}\left[Y_{0, n, \delta}\right]^{\mathrm{vir}} .
$$

The Proposition follows.

Remark 2. In the non-convex case, much of this goes through but the perfect obstruction theories involved are no longer compatible along $\iota$.

Remark 3. Suppose now that $X$ is a smooth orbifold and that $E \rightarrow X$ is a line bundle on $X$ that satisfies $(*)$. A straightforward argument involving orbifold RiemannRoch [1, Section 7] shows that $E$ is convex if and only if $E$ is the pullback of a line bundle on the coarse moduli space of $X$. 


\section{Conclusion}

We have seen that the Quantum Lefschetz Hyperplane Principle can fail for orbifold complete intersections, in cases where the bundle defining the complete intersection is non-convex. Thus at the moment we lack tools to prove mirror theorems for such complete intersections, even when the ambient orbifold is toric. A positivity condition alone $(*)$ is not enough to force convexity: it is necessary also for the bundle involved to be the pullback of a bundle on the coarse moduli space. This latter condition is very restrictive, and so "most" bundles on orbifolds are not convex.

Despite the examples in this paper one may still hope that, under some mild conditions, genus-zero Gromov-Witten invariants of orbifold complete intersections coincide with appropriate twisted Gromov-Witten invariants. For example, the equivariant-Euler twisted I-function $I^{\mathrm{tw}}(t, z)$ in [4, Theorem 4.8] admits a nonequivariant limit when the bundle $E$ and the parameter $t$ involved satisfy certain mild conditions [4, Corollary 5.1]. This is surprising, because the conditions there do not imply convexity. So one can hope that the twisted I-function still calculates the genuine invariants in such cases. (In the examples in this paper, the relevant twisted I-function does not admit a non-equivariant limit.) For example, Guest-Sakai computed the small quantum cohomology of a degree 3 hypersurface in $\mathrm{P}(1,1,1,2)$ from the differential equation satisfied by the twisted I-function [9], showing that the result coincides with Corti's geometric calculation.

Establishing the relationship between Gromov-Witten invariants of orbifold complete intersections and twisted Gromov-Witten invariants will require new methods. In the case of positive, non-convex bundles on orbifolds, the geometry involved is very similar to that which occurs when studying higher-genus stable maps to hypersurfaces in smooth varieties. Zinger and his co-workers [15,21] and Chang and Li [3] have made significant progress in this area recently, and it will be interesting to see if their techniques shed light on the genus-zero orbifold case too.

\section{Acknowledgments}

The authors thank Huai-Liang Chang, Alessio Corti, Behrang Noohi, and Michael Rose for useful conversations. This research is supported by a Royal Society University Research Fellowship (TC), European Research Council Starting Grant 240123GWT, an NSERC Postdoctoral Fellowship (AG), and an NSF Postdoctoral Fellowship DMS-0902754 (PJ).

\section{References}

[1] D. Abramovich, T. Graber, and A. Vistoli, Gromov-Witten theory of Deligne-Mumford stacks, Amer. J. Math. 130(5), (2008), 1337-1398.

[2] K. Behrend and B. Fantechi, The intrinsic normal cone, Invent. Math. 128(1), (1997), 45-88.

[3] H.-L. Chang and J. Li, Gromov-Witten invariants of stable maps with fields (2011), arXiv:1101.0914 [math.AG].

[4] T. Coates, A. Corti, H. Iritani, and H.-H. Tseng, Computing genus-zero twisted Gromov-Witten invariants, Duke Math. J. 147(3), (2009), 377-438.

[5] D. A. Cox, S. Katz, and Y.-P. Lee, Virtual fundamental classes of zero loci, in Advances in algebraic geometry motivated by physics (Lowell, MA, 2000), Vol. 276 of Contemporary Mathematics, 157-166, Amer. Math. Soc., Providence, RI (2001). 
[6] A. Givental, Equivariant Gromov-Witten invariants, Int. Math. Res. Notices (13) (1996), 613-663.

[7] - A mirror theorem for toric complete intersections, in Topological field theory, primitive forms and related topics (Kyoto, 1996), Vol. 160 of Progress in Mathematics, 141-175, Birkhäuser Boston, Boston, MA 1998.

[8] - Elliptic Gromov-Witten invariants and the generalised mirror conjecture, in integrable systems and algebraic geometry (Kobe/Kyoto, 1997), 107-155, World Science Publ., River Edge, NJ 1998.

[9] M. Guest and H. Sakai, Orbifold quantum D-modules associated to weighted projective spaces (2008). arXiv:0810.4236 [math.AG].

[10] P. Johnson, R. Pandharipande, and H.-H. Tseng, Abelian Hurwitz-Hodge integrals, Michigan Math. J. 60(1), (2011), 171-198.

[11] B. Kim, A. Kresch, and T. Pantev, Functoriality in intersection theory and a conjecture of Cox, Katz, and Lee, J. Pure Appl. Algebra 179(1-2), (2003), 127-136.

[12] M. Kontsevich and Y. Manin, Gromov-Witten classes, quantum cohomology, and enumerative geometry, Comm. Math. Phys. 164(3), (1994), 525-562.

[13] G. Laumon and L. Moret-Bailly, Champs algébriques, Vol. 39 of Ergebnisse der Mathematik und ihrer Grenzgebiete. 3. Folge. A Series of Modern Surveys in Mathematics [Results in Mathematics and Related Areas. 3rd Series. A Series of Modern Surveys in Mathematics], Springer-Verlag, Berlin (2000), ISBN 3-540-65761-4.

[14] J. Li and G. Tian, Virtual moduli cycles and Gromov-Witten invariants of algebraic varieties, J. Amer. Math. Soc. 11(1), (1998), 119-174.

[15] J. Li and A. Zinger, On the genus-one Gromov-Witten invariants of complete intersections, J. Differential Geom. 82(3), (2009), 641-690.

[16] B. H. Lian, K. Liu, and S.-T. Yau, Mirror principle. I, Asian J. Math. 1(4), (1997), 729-763.

[17] - Mirror principle. II, Asian J. Math. 3(1), (1999), 109-146. Sir Michael Atiyah: a great mathematician of the twentieth century.

[18] —, Mirror principle. III, Asian J. Math. 3(4), (1999), 771-800.

[19] I. Moerdijk, Introduction to the language of stacks and gerbes (2002). arXiv:math/0212266v1 [math.AT].

[20] J. Ross and R. Thomas, Weighted projective embeddings, stability of orbifolds, and constant scalar curvature Kähler metrics, J. Differential Geom. 88(1), (2011), 109-159.

[21] R. Vakil and A. Zinger, A desingularization of the main component of the moduli space of genus-one stable maps into $\mathbb{P}^{n}$, Geom. Topol. 12(1), (2008), 1-95.

Department of Mathematics, Imperial College London, 180 Queen's Gate, London SW7 2AZ, UK

E-mail address: t.coates@imperial.ac.uk

Department of Mathematics, University of Maryland, 1301 Mathematics Building, College Park, MD 20742-4015, USA

E-mail address: amingh@math.umd.edu

Department of Mathematics, Graduate School of Science, Kyoto University, OiwakeCho, Kitashirakawa, SAKyo-Ku, KyOto 606-8502, Japan

E-mail address: iritani@math.kyoto-u.ac.jp

Department of Mathematics, Imperial College London, 180 Queen's Gate, London SW7 2AZ, UK

E-mail address: y.jiang@imperial.ac.uk

Mathematics Department, Columbia University, Room 509, MC 4406, 2990 Broadway, New York, NY 10027, USA

E-mail address: pjohnson@math.columbia.edu

Institut für Mathematik, Humboldt Universität, Berlin, Germany

E-mail address: manolach@mathematik.hu-berlin.de 
